\title{
GENETIC ALGORITHM ASSISTED ADAPTIVE BEAMFORMING
}

\author{
M. Vitale, G. Vesentini, N.N. Ahmad, L. Hanzo \\ Dept. of ECS Univ. of Southampton, SO17 1BJ, UK. \\ Tel: +44-703-593 125, Fax: +44-703-594 508 \\ chandy@tiscalinet.it, glvese@libero.it, lh@ecs.soton.ac.uk \\ http: //www-mobile.ecs.soton.ac.uk
}

\begin{abstract}
Genetic Algorithm (GA) assisted beamforming techniques are proposed as an alternative to conventional beamforming algorithms. The design of the corresponding GAs is highlighted and the achievable performance is characterised in terms of the Signal-to-Interference Ratio (SIR) and the Signal-to-Interference plus Noise Ratio (SINR). It is demonstrated that an attractive SINR versus complexity trade-off is achievable by the proposed GA-assisted beamformers, provided that the GA parameters are appropriately chosen.
\end{abstract}

\section{INTRODUCTION}

Adaptive beamforming [1,2] is capable of separating signals transmitted in the same frequency band, provided that they arrive from different angles, i.e. they are separated in the spatial domain. This facilitates the separation of the desired signal from the interfering signals. The beamforming process appropriately combines the signals received by the different elements of an antenna array in order to form a single output. An adaptive antenna array is capable of optimising the beamforming pattern by adjusting the array weights such that the specific objective function used - for example the Signal-to-Interference plus Noise Ratio (SINR) is optimised. The specific choice of the adaptive beamforming algorithm determines the speed of convergence, the hardware complexity required for implementing the algorithm and the overall performance of a system.

The properties of the antenna array may be varied over time in order to optimise the system's performance for satisfying different optimisation criteria.These optimisation criteria may include minimizing the Mean-Square Error (MSE) between the desired and the actual array output, maximizing the array's output power, maximizing the Signal to Noise Ratio (SNR), minimizing the effect of interference or maximizing the SINR, etc. Depending on the array's operational environment, its performance optimisation metric and control algorithm may be varied in order to provide the best quality of service for the network users.

The optimisation of the antenna array pattern is typically subject to a range of constraints and the classic methods of array weight optimisation [1] may get trapped in local minima, resulting in a suboptimum beamforming performance. Consequently,

VTC 2002, Vancouver,Canada

The financial support of the following organisations is gratefully acknowledged: European Union; EPSRC, Swindon, UK; heuristic optimisation algorithms, based on random search strategies have often been employed in this field $[1,4-6,10]$.

In this paper, a Genetic Algorithm (GA) is developed for solving the beamforming weight optimisation problem. The concept of a GA is based on optimisation search strategies relying on the Darwinian principle of natural selection and evolution [9]. In the beamforming context the optimisation process involves a set of potential array vector solutions, which are referred to as individuals in GA parlance that are gradually - but not monotonously - improved with the aid of genetic operations. The GA-assisted optimisation process requires the definition of a so-called fitness function, which is evaluated for each individual throughout the evolutionary optimisation process exploiting the survival of the 'fit individuals' in the natural selection. The aim of our study is to determine the weight values of an antenna array by using GAs.

In Section 2 we will investigate the performance potential of a state-of-the-art GA, seeking the configurations optimising the performance in terms of co-channel interference reduction, i.e. SIR and SINR maximization. Throughout our investigations we always relate the achievable performance to the complexity required. Novel GA assisted techniques designed for enhancing the achievable performance are presented in Section 3, which are capable of expediting the search, while reducing the complexity necessitated. Finally, in Section 4 a GA based scheme is employed in conjunction with a spatial reference technique for more effectively exploiting a range of new GA features.

\section{STATE-OF-THE-ART IN GA-ASSISTED BEAMFORMING}

\subsection{GA-Aided Adaptive Beamforming}

In order to introduce the family of GAs cast in the context of beamforming problems, we will highlight the model also often used in the studies reported in the literature $[3,5,10]$. Specifically, we consider a linear array of $L$ omnidirectional elements placed in the far field of $M$ uncorrelated point sources, separated by a distance of $d=\lambda / 2$, where $\lambda$ is the wave-length of the sources. Thus, the GA is employed for optimising a set of $L$ complex array weights on the basis of the information contained in the so-called reference sequence [1] of each user. This strategy is referred to as the Temporal Reference Technique (TRT).

All examples presented here will refer to a scenario associated with a two-element antenna array and one interferer located at 27 degrees with respect to the desired user. In general GA based optimisation has to be able to perform six basic tasks [8]: 
1. Encode the potential array weight solutions with the aid of the genes of the GA;

2. Create a string of the GA's genes for the sake of forming a chromosome;

3. Create an initial population of GA-based solutions;

4. Evaluate and assign fitness values to individuals in the population of the GA;

5. Perform reproduction with the aid of the fitness-proportionate selection of individuals from the population for creating the next generation of high average fitness individuals;

6. Perform mutation of the individuals of the new generation by slightly perturbing the individuals for the sake of promoting a higher diversity of solutions in an effort to avoid getting trapped in local minima.

Each weight of the antenna array is represented by a string of bits, where half the bits corresponds to the real part and the other half to the imaginary part of the array weight concerned. This representation assists us in creating a genetic model for the weight adaptation problem. Hence each complex array weight has two genes, namely a real and an imaginary gene, which jointly constitute a chromosome. The set of chromosomes represents an individual, which corresponds to the $L$-dimensional complex weight vector of the $L$ elements of the array. The totality of the individuals, stored in a given generation of weight vectors, is referred to as the population.

The number of bits we used for representing each gene was a variable. It is important to be able to vary the number of bits per gene, since different optimisation problems may impose different constraints in terms of the target performance and the affordable complexity. The decision variables used in our GA assisted beamformer were quantized to a given number of bits, although the weights of the antenna array to be calculated are continuous valued. We opted for the straightforward binary coded decimal (BCD) representation of the weight values.

The optimisation criterion we will use for adjusting the weights is the minimisation of the MSE between the array output and the reference signal [1]. In order to evaluate the fitness values of each chromosome, the heuristic objective function of $F_{n}=1 /\left(1+E_{n}\right)$ has been used, where $F_{n}$ is the fitness value of the $n$th individual and $E_{n}=\left|r-\mathbf{w}^{H} \mathbf{x}\right|^{2}$ is the error term associated with the $n$th array weight. Finally, $r$ is the reference sequence, $\mathbf{w}^{H}$ is the Hermitian of the array weight vector and $\mathbf{x}$ is the received signal vector. Note that the choice of this objective function is somewhat ad hoc and it is based on the requirement of ensuring a reciprocal relationship between the error term $E_{n}$ and the fitness $F_{n}$, while also avoiding having an infinite fitness, when we have $E_{n}=0$. This results in fitness values between zero and unity.

\subsection{Simulation Results}

The main objective of our study is to find the set of GA parameters, which result in the highest possible GA performance. This task is extremely important, since different GAs having the same complexity may have dramatically different performances. We will commence our study by investigating one of the basic GA configurations known from the literature [6,7], which is shown in Table 1.

Fig. 1 (a) shows the achievable SIR performance versus the SNR of the GA-assisted beamformer characterised in Table 1 for

\begin{tabular}{|l|r|}
\hline Bits per gene & 10 \\
\hline Selection type & Roulette Selection \\
\hline Crossover type & Uniform \\
\hline Crossover probability & 0.9 \\
\hline Scaling & No Scaling \\
\hline Mutation type & Bit inversion \\
\hline Mutation probability & 0.1 \\
\hline Elitism & Performed \\
\hline
\end{tabular}

Table 1: GA parameters

both a high- and a low-complexity scenario associated with a population size of 60 and a total of 60 generations, as well as a population size of 200 and 600 generations, respectively. The complexity of the associated search may be characterised with the aid of the number of objective function evaluations, which is given by the product of the number of the generations and the population size. As expected, better SIR values are achieved, when increasing the affordable complexity. Furthermore, the sensitivity of the SIR values with respect to the number of bits used for representing the array weights is also shown in Fig. 1 (a).

The saturation of the SIR versus SNR=INR curves seen in Fig. 1 (a) at high SNR values suggests the presence of premature convergence to local minima, especially when the search-space is not fully explored due to the relatively low affordable complexity. In order to exploit the full potential of the GA-based optimisation algorithm - given a certain affordable complexity - the best possible GA configuration has to be found with the aim of preserving sufficient diversity for exploring the entire array-weight search space.

More explicitly, our aim is to configure the GA for exploring the entire space of the array weight solutions, preventing premature convergence, when relatively high-fitness but nonetheless suboptimum individuals are found. In other words, we will attempt to render the GA "more doubtful" about the reliability of the first results emerging from the search, in order to ensure that other potential solutions will not be neglected. Naturally, avoiding the premature convergence will considerably reduce the rate of convergence. Hence, our aim is to find an attractive trade-off between the rate of convergence and the achievable SIR.

We used elitism for replacing the low-fitness offspring of the new generation by the highest-fitness individuals of the previous generation. Elitism is efficient in promoting convergence, but hence it also may in fact encourage premature convergence without exploring the entire search space with sufficient grade of diversity for the solutions. Hence in our investigations we achieved the required population diversity by increasing the mutation probability from 0.05 to 0.1. Additionally, we also used the so-called Stochastic Remainder Selection technique (SRS) [9] for giving also the relatively low-fitness individuals a higher chance to reproduce and then employed the technique of Power Scaling (PS) for expediting the search [9]. The employment of a single point crossover was found to be more effective than that of a multi-point crossover zone, when combined with binary mutation, i.e. with bitinversion applied to binary encoded variables. The specific choice and combination of the above-mentioned GA parameters is justified with the aid of the results seen in Fig. 2, portraying our comparison between the standard suboptimum GA configuration and 

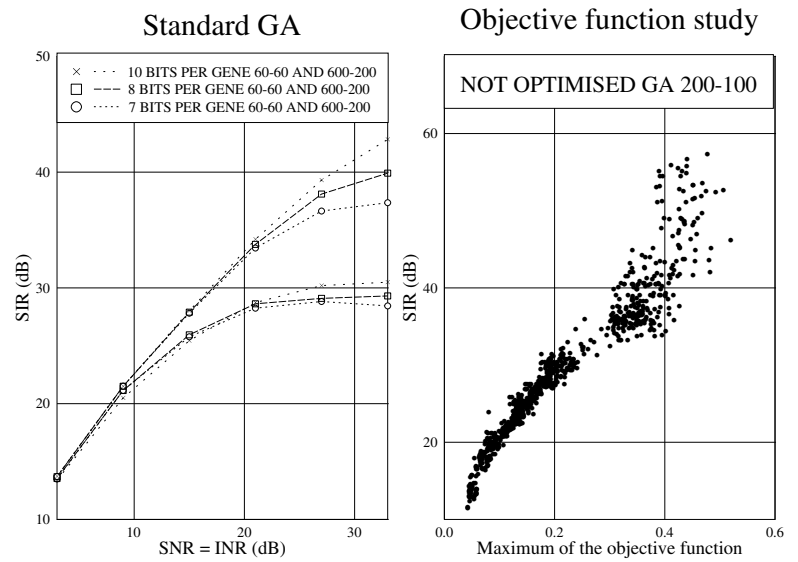

Figure 1: (a) The interference rejection expressed in terms of SIR versus SNR = INR using 7-, 8- and 10-bit encoding for each gene. The GA configuration was as follows. Selection: roulette based; crossover type: uniform; crossover probability: 0.9 ; mutation probability: 0.05 ; no scaling, elitism. The number of generations and population size was varied for the two-element antenna array studied, using a reference sequence length of 16 bits and an element spacing of $\lambda / 2$ assuming SNR $=$ INR. The source was at 0 degrees and the interferer at 27 degrees. The results were averaged over $10000 \mathrm{GA}$ runs, when communicating over a Gaussian channel.

(b) Scatter diagram of the SIR versus the fitness values of the best individuals of each run using a GA configured as in a).

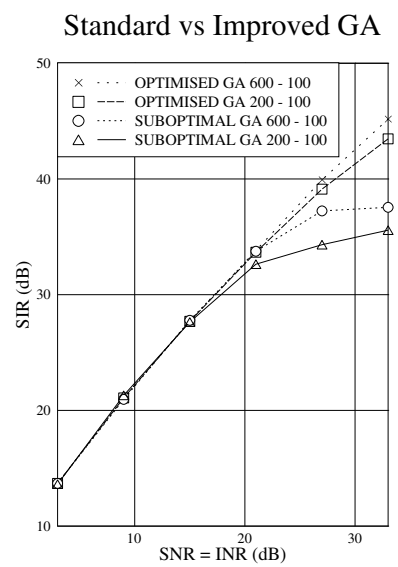

Figure 2: The interference rejection achieved expressed in terms of the SIR versus SNR = INR using 10-bit encoding for each gene in the improved optimised GA configuration. (Selection: stochastic remainder; crossover type: single point; crossover probability: 0.9 ; mutation probability: 0.1 ; power scaling with $\sigma=1.5$ ; elitism). The number of generations and the population size was varied for a two-element antenna array, using a reference sequence length of 16 bits and an element spacing of $\lambda / 2$ at $\mathrm{SNR}=\mathrm{INR}$. The source was at 0 degrees and the interferer at 27 degrees. The results were averaged over $10000 \mathrm{GA}$ runs, when communicating over a Gaussian channel. the above-mentioned improved GA configuration. It is interesting to note that despite its approximately $8 \mathrm{~dB}$ higher SIR performance, the improved GA-based beamformer has the same complexity expressed in terms of the product of the number of generations and the population size, as standard suboptimum scheme.

\section{ADAPTIVE GA}

\subsection{Developments in Genetic Algorithms}

In this section we set out to further reduce the complexity of the algorithm expressed as the product of the population size and the number of generations, while mantaining the same performance as in Fig. 2. As this stage we invoked a number of further GA operations, which are listed below:

- Weighted mutation was proposed for binary enviroments, where the assignment of different mutation probabilities depends on the significance of each bit of the binary-coded array weight. The less significant bits of each gene are assigned a higher mutation probability. This confines the effects of the mutation, focusing the search to the space surrounding the mutated individual. The aim of this technique is to prevent the search from diverging from the area that is likely to contain the optimum solution, which is typically activated only, when the GA succeded in identifying the broad area, where the optimal solution lies.

- Post selection retains those specific individuals for the next generation that are likely to achieve the best performance in the forthcoming generations. We note here that this principle is different from elitism, as it will highlighted below. The employment of this idea is based on two thresholds. If the fitness of the best individual of a generation is above a certain threshold, another threshold will be set to the value of $\left|F_{\text {average }}-F_{\text {best }}\right| / n$, where $F_{\text {average }}$ and $F_{\text {best }}$ represent the average fitness of the population and the fitness of the best individual, respectively, while $n$ is a parameter to be chosen depending on the specific problem considered. Each individual having a fitness below this second threshold is discarded and replaced in the following generation by a chromosome similar to the best one encountered so far. This is accomplished by applying a weighted mutation to the individual having the best fitness value so far throughout the search.

This technique "virtually" extends the population in the sense that it allows the GA to search in a wider space without increasing the complexity. The employment of post selection may potentially reduce the grade of population diversity, which has to be prevented by either mutation or scaling.

- Double search corresponds to an on-line re-initialization. The search invokes two GAs, where the second one uses a population generated by the results of the first one. Firstly, this technique aims for exploring the entire solution space, in order to locate the area for the optimal solution and secondly, for examining this 'promising' area in detail in order to find the best solution.

In our forthcoming elaborations we will employ a GA exhibiting the above features, which will be referred to as the "adaptive GA". 
Improved vs Adaptive GA

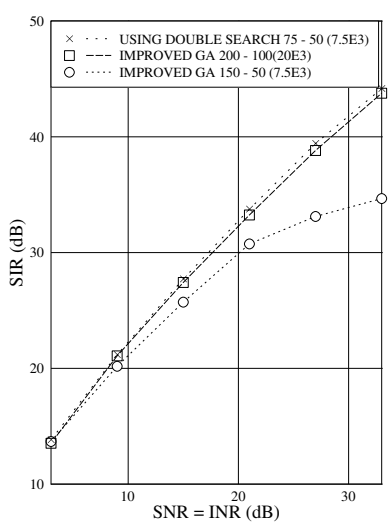

Objective function study

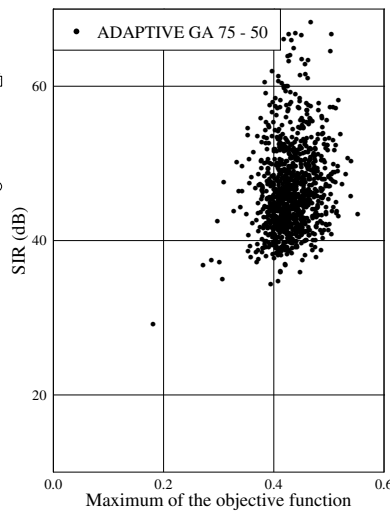

Figure 3: (a) The interference rejection achieved versus SNR = INR using 10-bit encoding of each gene in an improved GA configuration. (Selection: stochastic remainder, post selection with a threshold set at 0.22 and $n=10$; crossover type: single point; crossover probability: 0.9 ; mutation type: binary up to the fitness threshold of 0.22 , then weighted mutation; mutation probability: 0.1 ; power scaling with $\sigma=1$; elitism). The number of generations and population size was varied for a two-element antenna array, using a reference sequence length of 16 bits and an element spacing of $\lambda / 2$ at SNR $=$ INR. The source was at 0 degrees and the interferer was at 27 degrees. The result were averaged for $10000 \mathrm{GA}$ runs when comunicating over a Gaussian channel.

(b) Scatter diagram of the SIR versus the maximum fitness values of the individuals for each GA run using a GA configured as in a).

\subsection{Adaptive GA Performance}

The challenge in applying the features introduced in Section 3.1 is to appropriately set the point, where the GA has to change its search strategy. The results of our related investigations are shown in Fig. 3 (a) where it is clearly seen that for a normalised complexity of $7.5 \cdot 10^{3}$ expressed in terms of the product of the population size and the number of generations, the adaptive GA of Section 3.1 has a better SIR performance, than the improved GA of Fig. 2. For example, when considering Fig. 3 (a) at an SNR of $33 \mathrm{~dB}$ and at a normalised complexity product of $(150 \times 50)=7.5 \cdot 10^{3}$, the new features of Section 3.1 enhanced the SIR performance by about $10 \mathrm{dBs}$. In other words, the double-search based adaptive GA achieves the same performance as the improved of GA of Fig. 2, although the latter exhibits a normalised complexity of $200 \times 100$ $=2 \cdot 10^{4}$ in comparison to $75 \times 2 \times 50=7.5 \cdot 10^{3}$.

The efficiency of the double-search based adaptive GA in optimising the objective function is further characterised in Fig. 3 (b). From the comparison of Fig. 1 (b) and Fig. 3 (b) it becomes evident that the phenomenon of premature convergence has been eliminated, since most fitness values are above 0.3. Unfortunately, however, the SIR spread does not seize to constitute a serious drawback, which limits the achievable performance, indicating that sometimes a high objective function value leads to low SIR values. These findings indicate that a better objective function is required for further improving the achievable performance. Hence in the

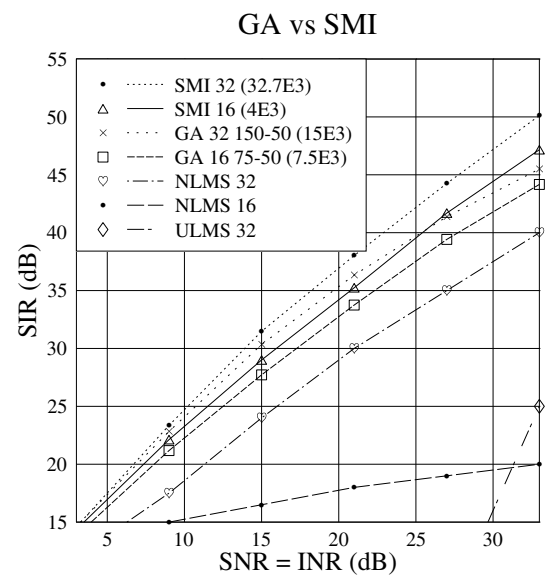

Figure 4: Comparison between the SIR performances of conventional benchmarker algorithms and that of a GA configuration. (Selection: stochastic remainder; crossover type: single point; crossover probability: 0.9 ; mutation probability: 0.1 ; power scaling with $\sigma=1$; elitism). The number of generations and the population size was varied for a two-element antenna array, using a reference sequence length of 16 and 32 bits and an element spacing of $\lambda / 2$ at $\mathrm{SNR}=\mathrm{INR}$. The source was at 0 degrees and the interferer was at 27 degrees. The results were averaged for 10000 GA runs, when comunicating over a Gaussian channel.

next section a more attractive optimisation criterion will be introduced.

\subsection{GA-Aided versus Conventional Beamformers}

Let us now benchmark the proposed GA-assisted beamformer against conventional techniques. The benchmarkers employed are the Sample Matrix Inversion (SMI), the Normalised Mean Squares Algorithm (NLMS) and the Unconstrained Least Mean Squares (ULMS) techniques [2].

In terms of their computational complexity, these algorithms exhibit rather dissimilar behaviours. The complexity of the SMI algorithm has a cubic relationship as a function of the number of antenna elements $L$, while the NLMS, the ULMS and GA-assisted beamformers exhibit a linear relationship as a function of the number of array elements, i.e. the number of array weights. Hence for a high number of array weights the SMI-aided beamformer exhibits a significantly higher complexity than that incurred by the others. In this context it is worth mentioning that while both the SMI and GA-assisted beamformers require the computation of the autocorrelation matrix of the received signal, only the SMI-aided beamformer has to invert the matrix. This results in an excessive complexity in case of a large autocorrelation matrix. On the other hand, the performance of the GA-aided beamformer appears to be closer to that of the SMI expressed in terms of the SIR, rather than to that of the NLMS and ULMS algorithms, as it is seen in Fig. 4.

\section{SIR OPTIMISATION IN ADAPTIVE BEAMFORMING}

In our previous investigations the MSE between the array output signal and the desired signal generated from the reference se- 
Objective function study

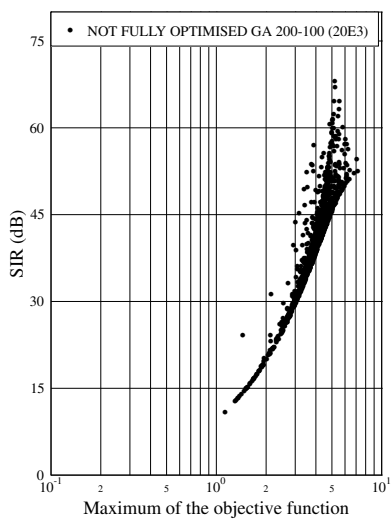

Direct SIR optimisation

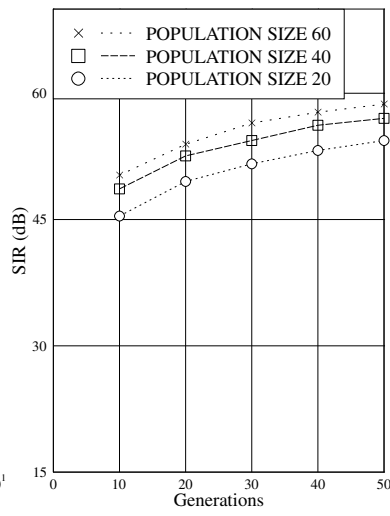

Figure 5: (a) Scatter diagram of the SIR versus the maximum fitness values of the individuals for each GA run, using a GA configurated as in b).

(b) The interference rejection achieved versus SNR = INR using 10-bit encoding for each gene in a rudimentary suboptimum GA - configuration. Selection: stochastic remainder; crossover type: single point; crossover probability: 0.9 ; mutation probability: 0.1 ; power scaling with $\sigma=1$; elitism; the angle of arrival was assumed to be estimated with a precision of 0.01 . The number of generations and population size was varied for a two element antenna array, using a reference sequence length of 16 bits and an element spacing of $\lambda / 2$ at SNR $=$ INR. The source was at 0 degrees and the interferer was at 27 degrees. The result were averaged for $10000 \mathrm{GA}$ runs when comunicating over a Gaussian channel.

quence was minimised. By contrast, our aim in this section is that of directly maximising the SIR. Hence we set the gradient of the SIR expression with respect to the array weights to zero and directly maximised the SIR by determining the weights with the aid of a GA. Our initial investigations suggested that in conjunction with this optimisation criterion interesting results emerge even when using unsophisticated GAs. Specifically, the SIR variance versus the objective function's maximum value seen in Fig. 5 (a) becomes narrower and this assists us in irradicating low SIR values, which were always experienced in conjunction with the MMSE criterion of Fig. 3. To elaborate a little further, a directly proportionate relationship between the fitness value and SIR is extremely desirable for ensuring that once the objective function is optimised by the GA, the optimum SIR value will be achieved. Again, Fig. 5 portrays the SIR values achieved by the GA specified in the figure caption, which is now consistently higher than the SIRs achieved by the benchmarkers of Fig. 4, whilst maintaining a reduced computational complexity. In this context we would like to note that this time the cost-function has been normalised in the post selection process, such that it may assume values in excess of unity.

\section{CONCLUSIONS AND CURRENT RESEARCH}

Various GA-assisted beamforming techniques have been proposed as an alternative to conventional beamforming algorithms. The design of the corresponding GAs was highlighted and their achiev- able performance was characterised in terms of both the SIR and the SINR. It was demonstrated that a potentially more attractive SINR versus complexity trade-off is achievable by the proposed GA-assisted beamformers. Further research is required for generalising these results to more complex beamformers and propagation scenarios.

Our current research is aimed at directly minimising the bit error rate of beam-forming assisted systems by employing the socalled the minimum bit error rate (MBER) approach. These techniques require an estimate of the probability density function (PDF) of the BER, which can be based on both block-adaptive and sampleby-sample adaptive approaches. Since the associated BER versus array-weight surface is significantly less well-behaved than the classic quadratic MSE error surface, the employment of the GAs investigated in this contribution is expected to be attractive in the context of directly optimising the BER. Consequently, our tentative results indicate that MBER beamforming is capable of providing significant performance gains in terms of a reduced BER over MMSE beamforming, while the employment of GAs has the promise of allowing us to avoid some of the associated deficiences of the BER optimisation problem.

\section{REFERENCES}

[1] J. Litva, T.K.Y. Lo, "Digital Beamforming in Wireless Communications" Artech House Publishers, 1996.

[2] J.S. Blogh, L. Hanzo: Third-Generation Systems and Intelligent Wireless Networking - Smart Antennas and Adaptive Modulation, John Wiley, 2002, p 408

[3] A. Tennant, M.M. Dawoud, and A.P. Anderson, "Array Pattern Nulling by Element Position Perturbations using GA " Electronics Letters, vol.30, pp.174-176, 1994.

[4] R.J. Mitchell, B. Chambers, and A.P. Anderson, "Array Pattern Synthesys in the Complex Plane Optimized by a GA," Electronics Letters, vol.32, pp. 1843-1845, 1996.

[5] R.L. Haupt, "Phase-only adaptive nulling with a GA, " IEEE Transactions on Antennas and Propagation., vol. 45, pp.10091015, 1997.

[6] K. Yen, L. Hanzo, "Hybrid Genetic Algorithm Based Detection Schemes for Synchronous CDMA Systems, "Vehicular Technology Conference 2000, Tokyo, Japan, 15-18 May 2000.

[7] D.S. Weile, E. Michielssen, "The Control of Adaptive Antenna Arrays With Genetic Algorithms Using Dominance and Diploidy, "IEEE Transactions on Antennas and Propagation, vol. 49, no. 10, pp. 1424-1433, October 2001.

[8] J.M. Johnson and Rahmat-Samii, "Genetic Algorithms in Engineering Electromagnetics, "IEEE Antennas and Propagation Magazine, vol. 39 , pp.7-21, 1997.

[9] D. E. Goldberg, "Genetic Algorithms in Search, Optimization, and Machine Learning, ” Addison-Wesley, 1989.

[10] M. Mitchell, "An Introduction to Genetic Algorithms, " Cambridge, MA: MIT Press, 1996. 